\title{
Análise dos parâmetros ultra-sonográficos empregados no diagnóstico da incontinência urinária de esforço
}

\author{
Evaluation of ultrasound parameters used as a diagnostic method for stress urinary incontinence
}

Rejane Maria Ferlin ${ }^{1}$, Almir Antonio Urbanetz ${ }^{2}$, José Gastão Rocha de Carvalho ${ }^{3}$, Mário Eduardo Rebolho ${ }^{4}$, Newton Sérgio de Carvalho ${ }^{5}$

\section{Resumo}

Objetivos: verificar a sensibilidade, especificidade e acurácia de medidas ultra-sonográficas da bexiga e uretra no diagnóstico da incontinência urinária de esforço (IUE). Métodos: o encurtamento da uretra, o abaixamento do colo vesical e a mudança da forma do trígono vesical, provocados pelo esforço miccional, foram medidos por ultra-sonografia transvaginal. A soma algébrica dos três indicadores foi utilizada como uma quarta medida para análise. A amostra era composta de 40 mulheres com IUE comparadas com 40 controles. Os casos diferiam dos controles com relação à idade, à paridade e ao número de gestações. Foram utilizados diversos pontos de corte para avaliar a sensibilidade, especificidade e acurácia diagnóstica do método. O diagnóstico urodinâmico da IUE e de controles serviu como padrão-ouro. Para a análise univariada dos dados, foram utilizados os testes $\chi^{2}$ de Yates e $\chi^{2}$ de Pearson. Resultados: no melhor ponto de corte para o abaixamento do colo vesical, a sensibilidade foi de $40 \%$, a especificidade de $72 \%$ e a acurácia de $57 \%$; no melhor ponto de corte para a medida do encurtamento da uretra, a sensibilidade foi de $40 \%$, a especificidade de $70 \%$ e a acurácia de $55 \%$; no melhor ponto de corte para a mudança da forma do trígono vesical, a sensibilidade foi de $58 \%$, a especificidade de $48 \%$ e a acurácia foi de 52\%; no melhor ponto de corte para soma das diferenças das três medidas, a sensibilidade do método foi de $32 \%$, a especificidade de $62 \%$ e a acurácia de $48 \%$. Conclusão: a ultra-sonografia transvaginal, no presente estudo, não se mostrou um método válido para o diagnóstico da IUE.

PALAVRAS-CHAVE: Incontinência urinária por estresse/terapia; Bexiga/ultrasonografia; Uretra/ultrasonografia; Colo do útero/ultrasonografia; Sensibilidade e especificidade

\section{Abstract}

Purpose: to verify the sensitivity, specificity and accuracy of some ultrasonographic measurements in the diagnosis of stress urinary incontinence (SUI). Methods: transvaginal ultrasound measurements of the bladder neck descent, urethral mobility and urethrovesical funneling caused by urination effort were performed in 40 women with SUI and in 40 women from a control group. Age, parity and the number of pregnancies were different in both groups. Several cut points were performed to evaluate the sensitivity, specificity and accuracy of these measurements as a diagnostic tool for SUI. The urodynamic results were used as gold standard. Univariated analysis was done using Yates $\chi^{2}$ Test and Pearson $\chi^{2}$ Test. Results: in the best cut point for bladder neck descent measurements, sensitivity was $40 \%$, specificity was $72 \%$ and accuracy was $57 \%$; in the best cut point for urethral mobility measurements, sensitivity was $40 \%$, specificity was $70 \%$ and accuracy was $55 \%$; in the best cut point for urethrovesical funneling measurements, sensitivity was $58 \%$, specificity was $48 \%$ and accuracy was $52 \%$; in the best cut point for the addition of the differences of these three measurements, sensitivity was

Trabalho realizado no Departamento de Medicina Interna e Departamento de Tocoginecologia da Universidade Federal do Paraná.

1 Médica ultra-sonografista da Maternidade do Hospital de Clínicas da Universidade Federal do Paraná - UFPR - Paraná (PR) - Brasil.

2 Professor do Departamento de Tocoginecologia da Universidade Federal do Paraná - UFPR - Paraná (PR) - Brasil.

3 Professor de Nefrologia e Chefe da Pós-Graduação do Departamento de Medicina Interna da Universidade Federal do Paraná - UFPR - Paraná (PR) - Brasil.

4 Professor e Chefe do Ambulatório de Uroginecologia do Hospital de Clínicas da Universidade Federal do Paraná - UFPR - Paraná (PR) - Brasil.

5 Professor do Departamento de Tocoginecologia da Universidade Federal do Paraná - UFPR - Paraná (PR) - Brasil.

Correspondência: Rejane Maria Ferlin

Rua Buenos Aires, 658, apto. 1702 -80250-070 - Curitiba - PR - e-mail: ecoclinica@uol.com.br 
$32 \%$, specificity was $62 \%$ and accuracy was $48 \%$. Conclusion: vaginal ultrasonography was not a valid diagnostic method for stress urinary incontinence in the present study.

KEYWORDS: Urinary incontinence, stress/therapy; Bladder/ultrasonografia; Urethra/ultrasonography; Cervix uteri/ ultrasonography; Sensitivity and specificity

\section{Introdução}

Incontinência urinária, pela padronização proposta pela Sociedade Internacional de Continência (ICS), é conceituada como qualquer perda involuntária de urina ${ }^{1}$. A incontinência urinária chamada "de esforço" (IUE) é definida como toda perda involuntária de urina, decorrente de esforço, exercício, espirro ou tosse. Do ponto de vista urodinâmico, a IUE é conceituada como a perda involuntária de urina através de canal uretral integro, quando a pressão vesical excede a pressão uretral máxima, na ausência de atividade do músculo detrusor ${ }^{1,2}$.

A IUE é importante porque afeta negativamente a qualidade de vida, sendo elemento gerador de exclusão social e interferindo na saúde física e mental ${ }^{3,4}$. Entretanto, apenas 59\% das mulheres procuram ajuda, pelo fato de não considerarem que o sintoma merece atenção médica ou, por outro lado, por considerarem o sintoma como parte do processo normal de envelhecimento ${ }^{5}$.

A avaliação inadequada e o diagnóstico incorreto da etiologia da incontinência urinária têm múltiplas conseqüências, sendo a mais séria a indicação de cirurgias inapropriadas ou mesmo desnecessárias. Procedimentos cirúrgicos recorrentes têm menores índices de sucesso, além de maior risco cirúrgico e maior taxa de complicações pós-operatórias ${ }^{4,5}$.

Para o diagnóstico da IUE, são necessários não apenas a adequada anamnese e o exame físico, mas também alguns exames complementares. Entre estes, diversos métodos de imagem têm sido descritos para o estudo da anatomia e função das estruturas responsáveis pelo suporte do colo vesical, como a uretrocistografia, a vídeouretrocistoscopia, a ultra-sonografia, a tomografia computadorizada e a ressonância magnética ${ }^{6,7}$. Alguns desses métodos são úteis, porém apresentam inconvenientes: a uretrocistografia é um método invasivo e pode ocasionar infecções urinárias; a tomografia e a ressonância magnética oferecem imagens de qualidade excepcional, porém têm o inconveniente do custo elevado ${ }^{7,8}$.

A ultra-sonografia tem sido utilizada para a investigação das estruturas do trato urinário baixo e, recentemente, foi incorporada ao estudo da IUE, não obstante ser uma prova complementar não in- dicada em todos os casos. Existem várias técnicas de exames ultra-sonográficos para avaliação do diagnóstico de IUE: transvaginal, transabdominal, transretal e transperineal ${ }^{7,8}$. Todos os métodos apresentam vantagens e limitações e ainda não se sabe qual é o mais preciso.

Com relação ao estudo da incontinência urinária feminina, a ultra-sonografia transvaginal foi eleita como conveniente por permitir a visualização simultânea da bexiga, do colo vesical, da uretra, da sinfise púbica e da vagina, num mesmo plano sagital, sem interferir com a mobilidade destas estruturas durante o esforço ${ }^{9}$.

Alterações anatômicas do colo vesical e da uretra proximal são consideradas como os componentes mais importantes da IUE. Em resposta ao aumento da pressão intra-abdominal, a hipermobilidade dessas estruturas devido ao suporte anatômico inadequado leva à perda involuntária de urina aos esforços $^{6,9}$. O grau de abaixamento do colo vesical tem sido utilizado para classificar a IUE e indicar a conduta terapêutica a ser adotada ${ }^{9}$. A mobilidade da uretra é também considerada um dos fatores mais relevantes no estudo de mulheres com IUE ${ }^{10}$.

Embora a mensuração ultra-sonográfica de diferentes ângulos entre o colo vesical e a sínfise púbica, durante repouso e esforço, tenham sido relatados na literatura, nenhuma dessas medidas mostrou sensibilidade, especificidade ou acurácia suficientes para ser usada rotineiramente na avaliação da paciente incontinente. A falta de padronização para os procedimentos e para o controle das variáveis individuais, as variações subjetivas do investigador na aplicação da técnica e o tamanho das amostras podem constituir os fatores responsáveis pelas divergências encontradas na literatura ${ }^{11}$.

Este estudo foi realizado com a finalidade de contribuir para a avaliação da eficácia da ultrasonografia como método diagnóstico alternativo e menos invasivo do abaixamento do colo vesical, encurtamento uretral ou mudança do trígono vesical, no repouso e no esforço, das pacientes com IUE.

\section{Métodos}

Realizou-se um estudo descritivo transversal para validação de teste diagnóstico num grupo de mulheres com e sem queixas de IUE, utilizando 
a urodinâmica como padrão ouro e a ecografia como teste.

Foram entrevistadas 900 mulheres atendidas no Ambulatório de Uroginecologia do Serviço de Ginecologia do Hospital de Clínicas da Universidade Federal do Paraná (UFPR), no período de 1996 a 2003. Os critérios para inclusão foram: mulheres com diagnóstico urodinâmico de IUE (casos) ou sem queixa clínica de perda urinária (controles) e que aceitaram participar voluntariamente do estudo. Foram excluidas as mulheres com cirurgias ginecológicas anteriores, história de doenças outras no trato urinário e em uso de medicação para IUE. Três pacientes foram excluídas da avaliação do abaixamento do colo vesical por terem expulsado o transdutor durante o esforço miccional, restando 77 pacientes para avaliação desse parâmetro. Todas as mulheres assinaram o Termo de Consentimento Livre e Esclarecido e o projeto foi aprovado pelo Comitê de Ética em Pesquisa da UFPR.

Considerou-se, para diagnóstico da IUE, a existência de quadro clínico caracterizado pela perda involuntária de urina aos esforços associada à tosse, ao espirro, ao riso e ao levantamento de peso, entre outros, confirmado pela anamnese e pelo teste urodinâmico e descartadas outras causas de perda urinária.

O teste urodinâmico obedeceu à padronização convencional e incluiu a cistometria, o estudo da pressão-fluxo e a fluxometria. O aparelho utilizado foi o Dynamed Uromaster 2.34, com medida simultânea de pressão vesical e pressão abdominal. Para medida da pressão vesical e para enchimento vesical, usou-se um cateter de urodinâmica $7 \mathrm{Fr}$. Para medida de pressão abdominal, usou-se sonda retal. O enchimento vesical foi realizado com soro fisiológico à temperatura de $37^{\circ} \mathrm{C}$, a uma velocidade de infusão de $35 \mathrm{~mL} / \mathrm{min}$.

O resultado do teste urodinâmico foi utilizado como padrão ouro para caracterizar a IUE e descartar outras alterações do trato urinário baixo que também podem levar à perda urinária. A aplicação do padrão ouro permitiu dividir as mulheres em duas categorias: com e sem IUE.

O teste ultra-sonográfico para diagnóstico da IUE foi avaliado com base em quatro medidas: abaixamento do colo vesical em relação ao púbis, comprimento da uretra, mudança de forma do trígono vesical e a medida resultante do somatório das anteriores.

Os exames foram realizados no Serviço de Ultra-sonografia da Maternidade do Hospital de Clinicas da UFPR, sempre pelo mesmo profissional. O aparelho utilizado foi o Toshiba-Tosbee, fabricado em Tokyo, Japão, com transdutor endovaginal de freqüência 7,5 MHz.

A realização dos exames ultra-sonográficos obedeceu à seguinte seqüência: as pacientes foram orientadas a ingerir aproximadamente $500 \mathrm{~mL}$ de água duas horas antes do exame. O volume prémiccional estabelecido foi de 250 a $500 \mathrm{~cm}^{3}$. O exame foi realizado com a paciente em posição de litotomia, sendo o transdutor revestido com preservativo e introduzido até aproximadamente dois centímetros do intróito vaginal. Após o aparecimento da imagem na tela, foram identificadas as seguintes estruturas: bexiga, colo vesical, sínfise púbica e uretra; após, procedeu-se às mensurações.

Para avaliação do abaixamento do colo vesical em relação à pube, as medidas foram realizadas traçando-se uma linha reta do bordo inferior da sínfise púbica até a junção uretrovesical. As medidas foram feitas em milimetros, em repouso e esforço miccional, tendo sido anotada a diferença. Utilizou-se o sinal (+) para as medidas acima da linha da sínfise púbica e o sinal (-) para as medidas abaixo do limite inferior da sinfise púbica. Utilizou-se o zero (0) quando não foram observadas diferenças (Figura 1).

O comprimento da uretra foi obtido considerando-se a porção visualizada na tela do monitor do aparelho de ultra-sonografia, desde a junção uretro-vesical até o bordo distal mais visivel. As

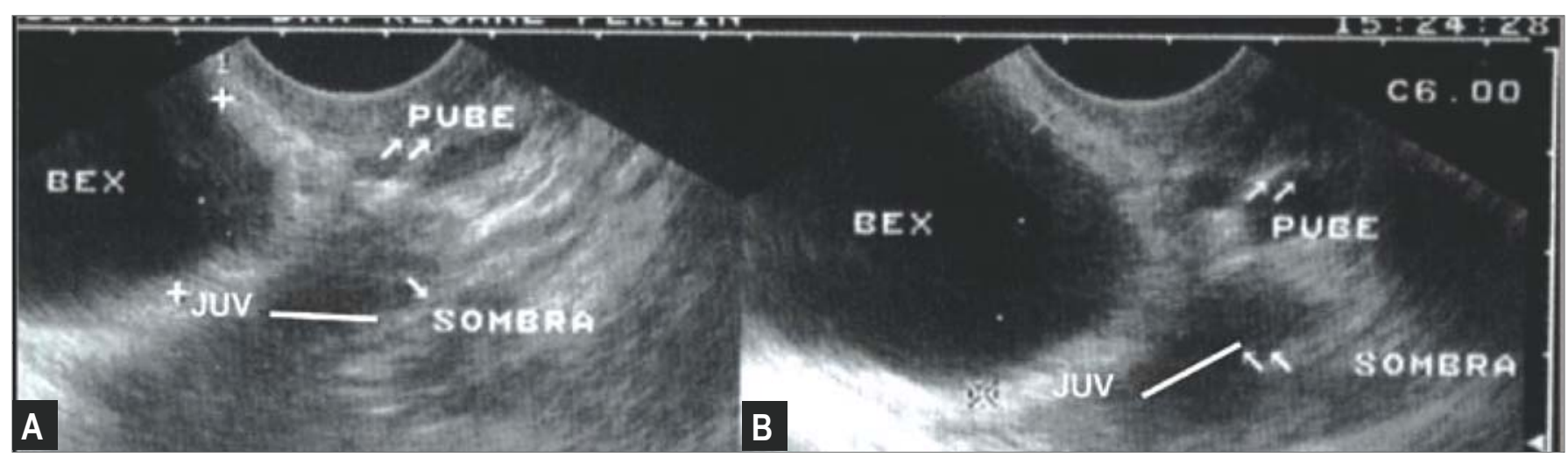

Figura 1 - Medidas do abaixamento do colo vesical em relação à pube, no repouso $(A)$ e no esforço $(B)$. 
medidas foram feitas em milímetros, com a paciente em repouso e em esforço miccional, tendo sido anotada a diferença (Figura 2).

Para avaliação da mudança de forma do trígono vesical (formato da bexiga); localizou-se o bordo vesical lateral esquerdo, desde a junção com o esfincter uretral interno, sendo então traçado um eixo paralelo e outro perpendicular ao bordo junto ao esfincter uretral interno. No ponto de interseção desses dois eixos foram traçadas duas linhas tangenciais oblíquas, para cima e para baixo, e estipulada a distância de $20 \mathrm{~mm}$ a partir do ponto inicial. Foi traçada, então, uma linha perpendicular unindo os dois pontos distais desses traçados de $20 \mathrm{~mm}$. Foi medida, no repouso e no esforço miccional, em milimetros, a distância entre a parede vesical superior e a inferior, tomando como base esta linha perpendicular e anotada a diferença. (Figura 3).

Depois de obtidas as medidas descritas, também foi considerada sua soma algébrica para avaliar a sua associação para o diagnóstico da IUE, resultando numa quarta medida. O objetivo pretendido era a criação de um novo parâmetro de avaliação diferente dos existentes e que pudesse, eventualmente, ser mais acurado que cada um dos indicadores isoladamente.
O instrumento de coleta de dados constituiuse de ficha estruturada de coleta, contendo dados de identificação da paciente (idade, peso, número de gestações, paridade, presença de IUE), data de realização do exame, resultados do exame clínico e do estudo urodinâmico e medições ultra-sonográficas dos parâmetros deste estudo.

A hipótese de normalidade da distribuição dos dados das diferentes amostras foi avaliada pelo teste de Shapiro-Wilk e pelo teste de KolmogorofSmirnof (pacote estatístico Statistica 6.0, StatSoft, Inc.). A comparação entre grupos não pareados com distribuições não-paramétricas foi feita pelo teste de Mann-Whitney. Distribuições normais foram encontradas nos dados referentes à mudança de forma do trígono vesical, nos grupos continente e incontinente, no qual foi usado o teste $t$ para amostras não-pareadas. Para grupos pareados com distribuições não-paramétricas, foi usado o teste de Wilcoxon, sendo que, somente na comparação entre a mudança da forma do trígono vesical, em repouso e durante o esforço, foi feito o teste $t$ para amostras pareadas. As amostras do abaixamento do colo vesical foram não-paramétricas, bem como a paridade em ambos os grupos e o número de gestações em mulheres continentes.

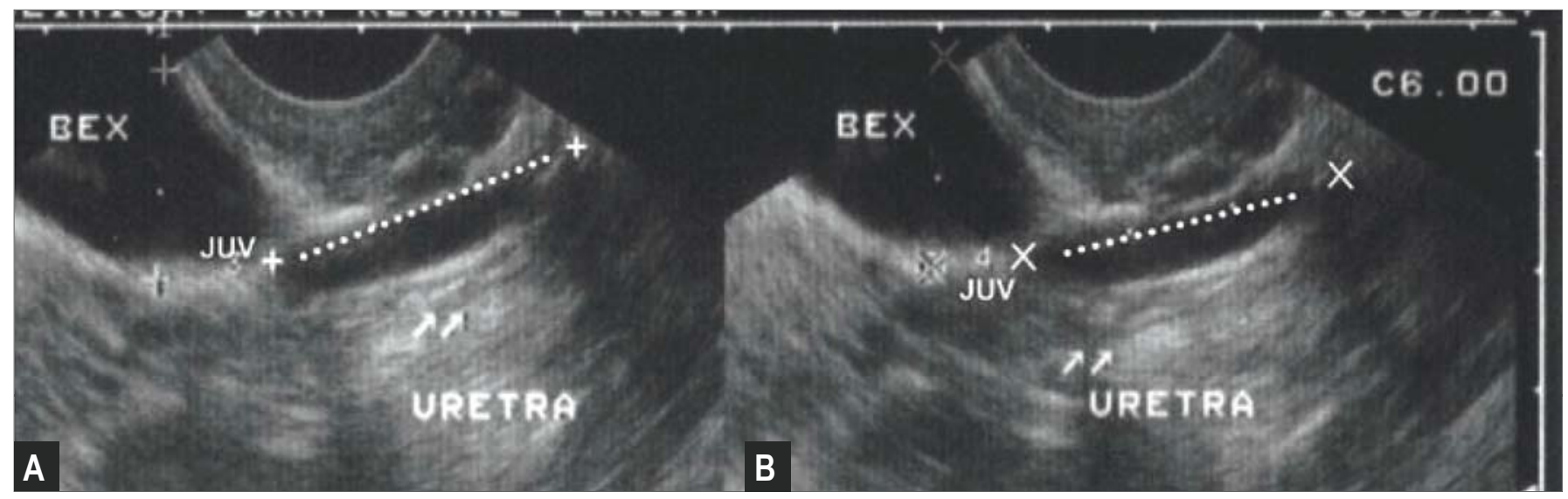

Figura 2 - Medida do comprimento da uretra, em repouso (A) e no esforço (B).

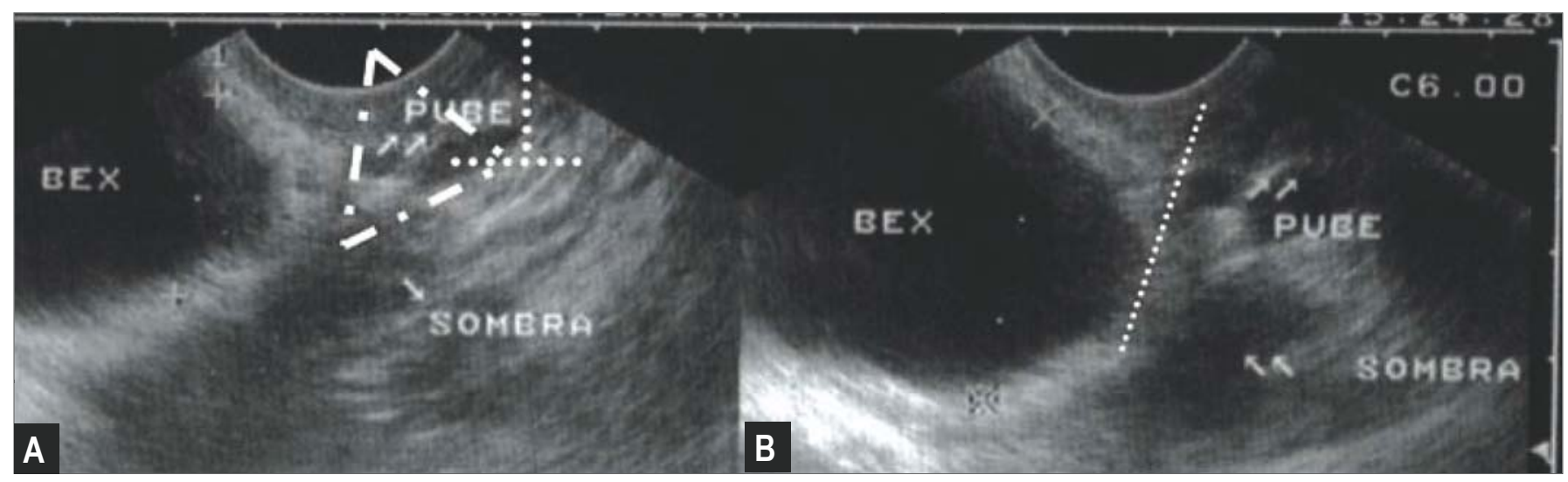

Figura 3 - Medida da mudança de forma do trígono vesical, em repouso (A) e no esforço (B).

Rev Bras Ginecol Obstet. 2006; 28(11): 633-42. 
Todos os resultados foram apresentados como média e desvio padrão e limites de confiança de -95 e $+95 \%$, com o valor de "p", sempre que indicado. Para a análise univariada dos dados, foram utilizados os testes $\chi^{2}$ de Yates e $\chi^{2}$ de Pearson. Foram utilizados diversos pontos de cortes para cada uma das medidas ultra-sonográficas e, para cada uma dessas medidas, procurou-se o ponto que melhor separasse as medidas dos casos daquelas dos controles. Essa medida foi utilizada como ponto de corte para dicotomizar os valores e avaliar a sensibilidade, especificidade e acurácia de cada teste. Posteriormente, procurou-se verificar a melhor combinação das três medidas que pudesse oferecer uma maior acurácia para o diagnóstico da IUE, tendo sido utilizada a soma algébrica da diferença dos três indicadores.

O grupo controle foi composto de mulheres sem queixa de incontinência urinária e diferia dos casos em termos das variáveis idade, paridade e número de gestações.

Observou-se que 68\% dos casos e apenas $25 \%$ dos controles tinham mais de 40 anos de idade, faixa acima da qual a literatura coloca como mais propensa ao início dos sintomas de IUE. A diferença entre os grupos foi significativa $(\mathrm{p}<0,001)$.

Quase a metade das mulheres continentes não teve nenhuma gestação, em comparação com apenas $5 \%$ das mulheres incontinentes. No outro extremo, $75 \%$ das mulheres incontinentes e $27 \%$ das mulheres continentes tinham três ou mais filhos $(p<0,004)$.
Com relação à paridade, apenas 5\% das mulheres incontinentes não tinham filhos e 65\% delas tinham três ou mais filhos $(p<0,001)$.

Com relação ao volume pré-miccional, não se observaram diferenças significativas entre as mulheres com incontinência urinária de esforço e as continentes $(p=0,83)$. Nas mulheres incontinentes, o volume pré-miccional variou entre 283 e $500 \mathrm{~cm}^{3}$, com média de $375,6 \mathrm{~cm}^{3}$ e mediana de $370,5 \mathrm{~cm}^{3}$. Nas mulheres continentes, o volume pré-miccional variou entre 250 e $500 \mathrm{~cm}^{3}$, com média de $370,7 \mathrm{~cm}^{3}$ e mediana de $395 \mathrm{~cm}^{3}$.

\section{Resultados}

As medidas obtidas por ultra-sonografia no encurtamento da uretra, na mudança da forma do trígono vesical, no abaixamento do colo vesical e no somatório dos três indicadores, em repouso e no esforço, não mostraram diferenças significativas entre os casos e os controles.

Todavia, houve diferenças significativas no grupo das mulheres incontinentes quanto às medidas obtidas em repouso e no esforço, quanto ao encurtamento da uretra (média no repouso $=26,7$ e no esforço $=21,1 ; \mathrm{p}<0,0001)$, da mudança do trígono vesical (média no repouso $=32,9$ e no esforço $=43,2 ; p<0,002)$ e do abaixamento do colo vesical (média no repouso=zero e no esforço $=3,9$; $\mathrm{p}<0,007$ ), como mostra a Tabela 1. Da mesma forma, houve diferenças significativas quanto às medidas obtidas em repouso e esforço nas mulheres continentes (Tabela 2).

Tabela 1 - Medidas ultra-sonográficas obtidas nas pacientes com incontinência urinária de esforço e respectiva análise estatística.

\begin{tabular}{|c|c|c|c|c|c|}
\hline Parâmetro & $\mathrm{p}$ & Média & Desvio-padrão & $\begin{array}{c}\text { LC } \\
-95 \%\end{array}$ & $\begin{array}{c}\text { LC } \\
+95 \%\end{array}$ \\
\hline Volume pré-miccional $\left(\mathrm{cm}^{3}\right)$ & $-\cdots$ & 375,6 & $\pm 69,6$ & 353,3 & 397,9 \\
\hline Encurtamento uretral em repouso (mm) & $<0,0001$ & 26,7 & $\pm 6,6$ & 24,6 & 28,8 \\
\hline Encurtamento uretral em esforço (mm) & $<0,0001$ & 21,1 & $\pm 7,3$ & 18,8 & 23,8 \\
\hline Mudança do trígono em repouso (mm) & $<0,002$ & 32,9 & $\pm 9,9$ & 29,7 & 36,7 \\
\hline Mudança do trígono em esforço (mm) & $<0,002$ & 43,2 & $\pm 10,5$ & 36,7 & 43,4 \\
\hline Abaixamento do colo vesical em repouso (mm) & $<0,0007$ & $-0,0$ & $\pm 8,3$ & $-2,8$ & 2,7 \\
\hline Abaixamento do colo vesical em esforço (mm) & $<0,0007$ & 3,9 & $\pm 5,2$ & $-0,6$ & 4,6 \\
\hline
\end{tabular}

$\mathrm{LC}=$ limite de confiança.

Tabela 2 - Medidas ultra-sonográficas obtidas no grupo controle e respectiva análise estatística.

\begin{tabular}{|c|c|c|c|c|c|}
\hline Parâmetro & $\mathrm{p}$ & Média & Desvio-padrão & $\begin{array}{c}\text { LC } \\
-95 \%\end{array}$ & $\begin{array}{c}\text { LC } \\
+95 \%\end{array}$ \\
\hline Volume pré-miccional $\left(\mathrm{cm}^{3}\right)$ & & 370,7 & $\pm 84,1$ & 343,8 & 397,6 \\
\hline Encurtamento uretral em repouso (mm) & $<0,00002$ & 26,0 & $\pm 4,6$ & 24,6 & 27,5 \\
\hline Encurtamento uretral em esforço (mm) & $<0,00002$ & 21,2 & $\pm 4,9$ & 19,7 & 22,8 \\
\hline Mudança do trígono em repouso (mm) & $<0,0002$ & 32,0 & $\pm 7,9$ & 29,5 & 34,5 \\
\hline Mudança do trígono em esforço (mm) & $<0,0002$ & 40,0 & $\pm 17,8$ & 37,5 & 48,9 \\
\hline Abaixamento do colo vesical em repouso (mm) & NS & $-1,7$ & $\pm 7,1$ & $-4,0$ & 0,5 \\
\hline Abaixamento do colo vesical em esforço $(\mathrm{mm})$ & NS & $-2,9$ & $\pm 6,9$ & $-5,2$ & $-0,6$ \\
\hline
\end{tabular}

NS = não significativo; $L C=$ limite de confiança. 
Considerado qualquer ponto de corte, a sensibilidade do método de abaixamento do colo vesical para o diagnóstico da IUE foi menor que $50 \%$ e a acurácia menor que $60 \%$. A especificidade ficou entre 62 e $72 \%$ no ponto de corte em que a sensibilidade ficou entre 40 e $49 \%$. O melhor ponto de corte foi $-7 \mathrm{~mm}$. Usando zero como ponto de corte, $84 \%$ das mulheres com IUE ficaram incluídas, mas também $98 \%$ das que não tinham a doença (especificidade de $2 \%$ ) (Tabela 3).

A acurácia da diferença do encurtamento da uretra nunca foi superior a $50 \%$ em qualquer ponto de corte. Os melhores pontos foram $\leq-7 \mathrm{~mm}$, com especificidade de $40 \%$ e especificidade de $70 \%$, e $\leq-5 \mathrm{~mm}$, com sensibilidade de $50 \%$ e especificidade de $55 \%$. A falta do encurtamento está colocada somente como referência, já que não tem validade para diagnóstico (Tabela 4).

A mudança da forma do trígono vesical também mostrou pouca acurácia e nunca foi superior a $52 \%$, no diagnóstico de IUE. No melhor ponto de corte $(\leq 8 \mathrm{~mm})$, a sensibilidade foi de $58 \%$ e a especificidade de $48 \%$ (Tabela 5).

Tabela 3 - Sensibilidade, especificidade e acurácia da diferença do abaixamento do colo vesical* pela ultra-sonografia para detecção da IUE. Análise dos diferentes pontos de corte $^{\star *}(n=77)^{\star * *}$.

\begin{tabular}{|c|c|c|c|c|c|}
\hline \multicolumn{3}{|c|}{ Diferença do relaxamento vesical } & \multirow{2}{*}{$\begin{array}{c}\text { Sensibilidade } \\
(\%)\end{array}$} & \multirow{2}{*}{$\begin{array}{c}\text { Especificidade } \\
(\%)\end{array}$} & \multirow{2}{*}{$\begin{array}{c}\text { Acurácia } \\
(\%)\end{array}$} \\
\hline Ponto de Corte & + & - & & & \\
\hline$\leq-11$ & 10 & 67 & 19 & 92 & 57 \\
\hline$\leq-9$ & 21 & 56 & 32 & 78 & 56 \\
\hline$\leq-7$ & 26 & 51 & 40 & 72 & 57 \\
\hline$\leq-5$ & 30 & 47 & 43 & 65 & 55 \\
\hline$\leq-3$ & 31 & 46 & 43 & 62 & 53 \\
\hline$\leq-2$ & 33 & 44 & 49 & 62 & 56 \\
\hline$\leq 0$ & 70 & 7 & 84 & 2 & 42 \\
\hline
\end{tabular}

*A diferença do abaixamento vesical foi obtida pela subtração das medidas sob esforço e sob repouso.

**As medidas são negativas pois, em geral, no esforço, são inferiores às do repouso.

***Três pacientes com IUE foram excluídas por impossibilidade de obtenção da imagem ultra-sonográfica para realização desta medida.

Tabela 4 - Sensibilidade, especificidade e acurácia da diferença do encurtamento da uretra* pela ultra-sonografia para detecção da IUE. Análise dos diferentes pontos de corte** ( $\mathrm{n}=80$ ).

\begin{tabular}{|c|c|c|c|c|c|}
\hline \multicolumn{3}{|c|}{ Diferença do encurtamento da uretra } & \multirow{2}{*}{$\begin{array}{c}\text { Sensibilidade } \\
(\%)\end{array}$} & \multirow{2}{*}{$\begin{array}{c}\text { Especificidade } \\
(\%)\end{array}$} & \multirow{2}{*}{$\begin{array}{c}\text { Acurácia } \\
(\%)\end{array}$} \\
\hline Ponto de Corte & + & - & & & \\
\hline$\leq-13$ & 9 & 71 & 12 & 90 & 51 \\
\hline$\leq-10$ & 15 & 65 & 22 & 85 & 54 \\
\hline$\leq-7$ & 28 & 52 & 40 & 70 & 55 \\
\hline$\leq-5$ & 38 & 42 & 50 & 55 & 52 \\
\hline$\leq-3$ & 51 & 29 & 65 & 38 & 51 \\
\hline$\leq-1$ & 66 & 14 & 82 & 18 & 50 \\
\hline 0 & 76 & 4 & 92 & 2 & 48 \\
\hline
\end{tabular}

${ }^{*}$ A diferença do encurtamento da uretra foi obtida pela subtração das medidas sob esforço e sob repouso.

${ }^{\star *}$ As medidas são negativas pois, em geral, no esforço, são inferiores às do repouso.

Tabela 5 - Sensibilidade, especificidade e acurácia da diferença* pela ultra-sonografia para detecção da IUE. Análise dos diferentes pontos de corte** $(n=80)$.

\begin{tabular}{|c|c|c|c|c|c|}
\hline \multicolumn{3}{|c|}{ Diferença da mudança da forma do trígono vesical } & \multirow{2}{*}{$\begin{array}{c}\text { Sensibilidade } \\
(\%)\end{array}$} & \multirow{2}{*}{$\begin{array}{c}\text { Especificidade } \\
(\%)\end{array}$} & \multirow{2}{*}{$\begin{array}{c}\text { Acurácia } \\
(\%)\end{array}$} \\
\hline Ponto de Corte & + & - & & & \\
\hline$\leq 0$ & 8 & 72 & 8 & 88 & 48 \\
\hline$\leq 2$ & 21 & 59 & 22 & 70 & 46 \\
\hline$\leq 4$ & 29 & 51 & 35 & 62 & 49 \\
\hline$\leq 6$ & 36 & 44 & 45 & 55 & 50 \\
\hline$\leq 8$ & 44 & 36 & 58 & 48 & 52 \\
\hline$\leq 12$ & 57 & 23 & 70 & 28 & 49 \\
\hline$\leq 16$ & 65 & 15 & 78 & 15 & 46 \\
\hline$\leq 22$ & 73 & 7 & 85 & 2 & 44 \\
\hline
\end{tabular}

${ }^{*}$ A diferença da mudança da forma do trígono foi obtida pela subtração das medidas sob esforço e sob repouso.

**As medidas são negativas pois, em geral, no esforço, são inferiores às do repouso. 
A sensibilidade, a especificidade e a acurácia da soma das diferenças das três medidas obtidas pelo exame ultra-sonográfico, com diversos pontos de corte para detecção da IUE mostrou que, no ponto de corte em que a sensibilidade do método foi superior a $80 \%$, a especificidade foi apenas $5 \%$ e a acurácia $44 \%$.

No melhor ponto de corte, mudança $\leq 8 \mathrm{~mm}$, a sensibilidade foi $32 \%$ e a especificidade $62 \%$ (Tabela 6).

Em nenhum ponto de corte a acurácia foi superior a $48 \%$, o que indica que a soma algébrica da diferença das medidas do abaixamento do colo vesical, mudança da forma do trígono vesical e encurtamento da uretra não é adequada para diagnóstico da incontinência urinária de esforço.

Considerando todas as medidas estudadas, verificou-se que a sensibilidade ficou entre $30 \mathrm{e}$ $60 \%$, a especificidade ficou entre 50 e $70 \%$ e a acurácia próxima a $50 \%$ em todos os casos.

Em relação ao abaixamento do colo vesical, o melhor ponto de corte foi $7 \mathrm{~mm}$, com acurácia de 57\%; com relação ao encurtamento da uretra, o melhor ponto de corte foi $7 \mathrm{~mm}$, com acurácia de 55\%; considerando-se a mudança da forma do trígono vesical, a maior acurácia foi de $52 \%$ no ponto de corte $8 \mathrm{~mm}$.

\section{Discussão}

Os resultados encontrados neste estudo indicam que a avaliação ultra-sonográfica não é um método eficaz para a detecção da IUE. As medidas do abaixamento do colo vesical, encurtamento da uretra e mudança da forma do trígono vesical e a soma algébrica dessas medidas, avaliadas pela ultra-sonografia, mostraram uma sensibilidade em torno de $50 \%$ e uma acurácia menor que $60 \%$, em qualquer ponto de corte considerado.

Esses resultados estão em concordância com os trabalhos realizados com amostras semelhantes, que concluíram que a ultra-sonografia não se constitui num método confiável para o diagnóstico da IUE ${ }^{12-14}$, e em discordância com outros trabalhos que encontraram diferenças significativas entre as mulheres continentes e incontinentes para todos os parâmetros estudados e consideram que a ultra-sonografia está em vias de tornar-se o método diagnóstico padrão em uroginecologia ${ }^{15-18}$. Outros autores, ainda, consideram que os dados obtidos através da ultra-sonografia, além de divergentes, não fornecem um suporte adequado para uma avaliação rigorosa do diagnóstico da IUE ${ }^{13,19,20}$.

Os estudos sobre a efetividade da ultra-sonografia para a avaliação do abaixamento do colo vesical no diagnóstico da IUE são controversos. Embora a mensuração de diferentes ângulos entre o colo vesical e sinfise púbica, durante repouso e esforço, tenha sido relatada, para a maioria dos autores, nenhuma dessas medidas mostrou sensibilidade, especificidade ou acurácia suficientes para ser usada rotineiramente na avaliação da paciente incontinente ${ }^{8,12,13}$. Neste estudo, a baixa especificidade da medida do abaixamento do colo vesical não permite recomendar a sua utilização para diagnóstico da IUE.

A ausência de validade diagnóstica para a IUE da diferença do encurtamento da uretra observada em nosso estudo está de acordo com os relatados por outros autores que, em estudos similares, não encontraram diferenças significativas neste parâmetro entre as mulheres continentes

Tabela 6 - Sensibilidade, especificidade e acurácia em diversos pontos de corte da soma algébrica das medidas do abaixamento do colo vesical, encurtamento da uretra e mudança da forma do trígono vesical para detecção da IUE (padrão-ouro), $n=77^{*}$.

\begin{tabular}{|c|c|c|c|c|c|}
\hline \multicolumn{3}{|c|}{ Soma das medidas } & \multirow{2}{*}{ Sensibilidade (\%) } & \multirow{2}{*}{ Especificidade (\%) } & \multirow{2}{*}{ Acurácia (\%) } \\
\hline Ponto de Corte & + & - & & & \\
\hline$\leq 2$ & 11 & 66 & 8 & 80 & 45 \\
\hline$\leq 4$ & 18 & 59 & 16 & 70 & 44 \\
\hline$\leq 6$ & 16 & 51 & 30 & 62 & 47 \\
\hline$\leq 8$ & 27 & 50 & 32 & 62 & 48 \\
\hline$\leq 10$ & 28 & 49 & 32 & 60 & 47 \\
\hline$\leq 12$ & 31 & 46 & 35 & 55 & 45 \\
\hline$\leq 14$ & 36 & 41 & 38 & 45 & 42 \\
\hline$\leq 16$ & 42 & 35 & 46 & 38 & 42 \\
\hline$\leq 18$ & 49 & 28 & 57 & 30 & 43 \\
\hline$\leq 20$ & 51 & 26 & 57 & 25 & 40 \\
\hline$\leq 22$ & 57 & 20 & 65 & 18 & 40 \\
\hline$\leq 24$ & 62 & 15 & 70 & 10 & 39 \\
\hline$\leq 34$ & 70 & 7 & 86 & 5 & 44 \\
\hline$\leq 44$ & 76 & 1 & 97 & 0 & 47 \\
\hline
\end{tabular}

*Três pacientes com IUE foram excluídas por impossibilidade de obtenção da imagem ultra-sonográfica para realização desta medida. 
e incontinentes e concluíram que, para um bom entendimento das condições normais e patológicas, seria necessário o desenvolvimento de um método ultra-sonográfico que permitisse a análise de toda a extensão da uretra ${ }^{12,14}$. Estes autores também concluíram que a hipermobilidade uretral pode ocorrer em pacientes sem queixas de incontinência e que sua presença não serve para indicar a existência ou a severidade da afecção. Outros estudos, entretanto, relatam que o sinal ultra-sonográfico mais comumente associado à incontinência urinária é o encurtamento da uretra proximal durante o extravasamento da urina e os autores introduziram o conceito de limiar de continência, definido como o equilibrio entre as forças de contenção e expulsão que atuam sobre a uretra, propondo um modelo teórico para a incontinência, no qual três estágios anatômicos hipotéticos estariam presentes: o primeiro estágio seria representado pelo fechamento uretral antes de atingido o limiar de continência; no segundo estágio, haveria um equilíbrio entre as forças de contenção e expulsão; no terceiro estágio, o aumento da pressão intra-abdominal determinaria a ruptura do equilíbrio entre as forças, o que resultaria em encurtamento da uretra e extravasamento de urina, sendo esse indicador considerado como um parâmetro ultra-sonográfico válido para o diagnóstico da IUE ${ }^{6,16,21}$.

A avaliação ultra-sonográfica da mudança da forma do trígono vesical também mostrou pouca acurácia para o diagnóstico da IUE. O motivo da escolha desta medida foi porque, ao realizarmos a medida do abaixamento do colo vesical, percebemos uma alteração da forma da bexiga (arredondamento do bordo lateral) que parecia ser significativa, o que não foi confirmado ao realizarem-se medidas para comprovar esta alteração. Não encontramos nenhum trabalho na literatura que avaliasse este parâmetro.

Além de avaliar as três medidas ecográficas isoladamente, também foi considerada a soma algébrica dessas mesmas medidas. O objetivo pretendido era a criação de um novo parâmetro de avaliação diferente dos existentes e que, ao somar as variações nos três parâmetros, pudesse, eventualmente, ser mais acurado que cada um dos indicadores isoladamente. Infelizmente, este novo indicador também se mostrou inapropriado para o diagnóstico da IUE, sem que possamos comparar nossos resultados com os dos outros pesquisadores, já que, na literatura mundial, observa-se apenas a descrição desses indicadores isoladamente. A justificativa da proposta da ultra-sonografia como método alternativo à radiografia e à vídeocistouretrografia são os custos menores do equipamento, ausência de radiação e por ser menos invasiva. Entretanto, a baixa sensibilidade e especificidade mostrada não só em nossos resultados, mas também na literatura, não favorecem sua utilização para este propósito. Os motivos da baixa acurácia do método podem estar relacionados a alguns problemas importantes para a utilização da ultra-sonografia como método de avaliação do trato urinário baixo, como a distorção nos resultados causada pelo uso do transdutor ou o problema da sombra acústica ${ }^{2,9,22,23}$.

Embora a distorção causada pelo uso do transdutor possa ser considerada e detectada, não foram encontradas evidências objetivas de que possa ser eliminada. Transdutores vaginais mais modernos são finos e dão uma boa visualização do trato urinário quando introduzidos a uma pequena distância no intróito vaginal, mas não se conhece como o transdutor, por si só, modifica a estrutura do trato urinário baixo. Outras possiveis explicações foram procuradas por alguns pesquisadores que avaliaram a influência do transdutor nos parâmetros urodinâmicos e nas medidas dos ângulos ou da mobilidade da uretra e da bexiga, observaram que sua introdução resulta no aumento da pressão máxima da uretra e concluíram que o seu uso resulta na compressão da uretra, aumento da pressão uretral e elevação vesical, o que pode influir não só na detecção da incontinência, bem como reduzir a severidade da incontinência detectada. Ainda, como a instabilidade do músculo detrusor é um dos fatores responsáveis pela incontinência urinária, a utilização do transdutor vaginal pode mascarar a avaliação dessa condição pelo aumento da pressão uretral sobre esta musculatura ${ }^{14,18,24}$.

O problema da sombra acústica da junção uretrovesical pela sínfise púbica foi descrito como outro fator limitante do uso da ultrasonografia para avaliação do abaixamento do colo vesical, o que ocorreu em uma das avaliações deste estudo ${ }^{25}$.

A inconsistência dos achados da literatura sugere que é necessário encontrar métodos ultrasonográficos mais acurados para documentar os suportes anatômicos das estruturas do trato urinário baixo ${ }^{9}$.

A reprodutibilidade de resultados de medidas ultra-sonográficas é deficiente devido às variações biológicas individuais de cada sujeito investigado e às variações subjetivas do investigador na aplicação da técnica. Constitui uma limitação do método o fato de as técnicas não serem padronizadas e introduzirem variações nos resultados ${ }^{18}$. Apesar de termos estabelecido uma padronização de técnica para realização de exames no presente estudo e de termos tentado seguir uma metodologia pré-estabelecida, há fatores que fogem ao nosso controle objetivo, os quais dizem respeito aos aspectos subjetivos de cada paciente, como a sua força exercida no momento da realização 
do exame, sua cooperação e sua participação durante o exame. Os testes ultra-sonográficos medem aspectos específicos do mecanismo de continência sob condições clínicas diferentes - como, por exemplo, a intensidade do esforço realizado individualmente no momento do exame, o que limita a comparação direta entre eles. Em nosso estudo, ao realizarmos as medidas do esforço miccional, a força realizada por algumas pacientes resultou na expulsão do transdutor da vagina e impediu a avaliação das medidas. Essas pacientes poderiam ter sido incluídas no estudo se tivessem efetuado menos força, do mesmo modo que outras poderiam ter sido excluídas se tivessem feito maior força, o que pode provocar um viés de seleção dificil de controlar.

A IUE é uma afecção de causa multifatorial: a idade, a paridade, a obesidade e as lacerações do assoalho pélvico causadas por trauma ou cirurgia estão entre os principais fatores desencadeantes. Também, o grau de severidade da incontinência é um aspecto extremamente variável. Na investigação ultra-sonográfica da IUE, as avaliações das medidas do abaixamento do colo vesical e do encurtamento da uretra são realizados com base em diferentes eixos e parâmetros e, em geral, em amostras pequenas.

Assim, a falta de padronização para os procedimentos e para controle das variáveis individuais, bem como o tamanho da amostra, podem constituir os fatores responsáveis pelas divergências encontradas na literatura ${ }^{17,26,27}$, o que dificulta a comparação entre os estudos e a obtenção de conclusões válidas que permitam a reprodutibilidade do método.

A ultra-sonografia é um método de diagnóstico excelente, que tem múltiplas aplicações nos diferentes ramos da medicina e resulta inapropriado colocar seu merecido prestígio em risco, tentando estender sua utilidade além dos limites em que é realmente indispensável. Com base nos resultados deste estudo, sugerimos que não desperdicem esforços e horas de uso de equipamentos custosos tentando realizar essa metodologia, que mostrou ser ineficaz.

Concluímos que o estudo ultra-sonográfico do abaixamento do colo vesical, da medida do encurtamento da uretra, da modificação da forma do trígono vesical e da medida resultante da soma algébrica desses parâmetros não se constitui num método adequado para o diagnóstico da incontinência urinária de esforço.

\section{Referências}

1. Abrams P, Cardozo L, Fall M, Griffiths D, Rosier $P$, Ulmsten $U$, et al. The standardisation of terminology of lower urinary tract function: report from the Standardisation Sub-comittee of the International Continence Society. Am J Ginecol Obstet. 2002;187(1):116-26.

2. Moreira SSF, Girão MJBC, Sartori MGF, Baracat EC, Lima GR. Mobilidade do colo vesical e avaliação funcional do assoalho pélvico em mulheres continentes e com incontinência urinária de esforço, consoante o estado hormonal. Rev Bras Ginecol Obstet. 2002;24(6):365-70.

3. Guarisi T, Pinto-Neto AM, Osis MJ, Pedro AO, CostaPaiva LHS, Faúndes A. Procura de serviço médico por mulheres com incontinência urinária. Rev Bras Ginecol Obstet. 2001;23(7):439-43.

4. Feldner Júnior PC, Bezerra LRPS, Girão MJBC, Castro RA, Sartori MGF, Baracat EC, et al. Correlação entre a pressão de perda à manobra de Valsalva e a pressão máxima de fechamento uretral com a história clínica em mulheres com incontinência urinária de esforço. Rev Bras Ginecol Obstet. 2002;24(7):433-8.

5. Broome BA. The impact of urinary incontinence on self-efficacy and quality of life. Health Qual Life Outcomes. 2003;1(1):35.

6. Mostwin JL, Genadry R, Saunders R, Yang A. Stress incontinence observed with real time sonography and dynamic fastscan magnetic resonance imaging: insights into pathophysiology. Scand J Urol Nephrol Suppl. 2001;(207):94-9; discussion 106-25.

7. Granados Loarca EA, Alcahe VR, de Leon López H, Echeverria Reyes J. Utilidad del ultrasonido perineal en la incontinencia de orina en la mujer. Arch Esp Urol. 1999;52(7):778-82.

8. Vicente Prados FJ, Cozar Olmo JM, Martinez Morcillo A, Espejo Maldonado E, Tallada BuñuelM. Incontinencia urinaria. Métodos para su evaluación y clasificación. Arch Esp Urol. 2002;55(9):1015-34.

9. Yalcin OT, Hassa H, Ozalp S. Effectiveness of ultrasonographic parameters for documenting the severity of anatomic stress incontinence. Acta Obstet Gynecol Scand. 2000;79(5):421-6.

10.Pregazzi R, Sartore A, Bortoli P, Grimaldi E, Troiano L, Guaschino S. Perineal ultrasound evaluation of urethral angle and bladder neck mobility in women with stress urinary incontinence. BJOG. 2002;109(7):821-7.

11.Dietz HP. Ultrasound imaging of the pelvic floor. Part I: two-dimensional aspects. Ultrasound Obstet Gynecol. 2004;23(1):80-92.

12.Otcenasek M, Halaska M, Krcmar M, Maresova D, Halaska MG. New approach to the urogynecological ultrasound examination. Eur J Obstet Gynecol Reprod Biol. 2002;103(1):72-4.

13.Betson LH, Siddiqui G, Bhatia NN. Intrinsic urethral sphincteric deficiency: critical analysis of various diagnostic modalities. Curr Opin Obstet Gynecol. 2003;15(5):411-20. 
14.Rovner ES, Wein AJ. Evaluation of lower urinary tract symptoms in females. Curr Opin Urol. 2003;13(4):273-8.

15.Novara G, Artibani W. Imaging for urinary incontinence: a contemporary perspective. Curr Opin Urol. 2006;16(4):219-23.

16.Tunn R, Goldammer K, Gauruder-Burmester A, Wildt $\mathrm{B}$, Beyersdorff D. Pathogenesis of urethral funneling in women with stress urinary incontinence assessed by introital ultrasound. Ultrasound Obstet Gynecol. 2005;26(3):287-92.

17.Sendag F, Vidinli H, Kazandi M, Itil IM, Askar N, Vidinli $\mathrm{B}$, et al. Role of perineal sonography in the evaluation of patients with stress urinary incontinence. Aust $\mathrm{N}$ Z J Obstet Gynaecol. 2003;43(1):54-7.

18.Alper T, Cetinkaya M, Okutgen S, Kokcu A, Malatyalioglu E. Evaluation of urethrovesical angle by ultrasound in women with and without urinary stress incontinence. Int Urogynecol $\mathrm{J}$ Pelvic Floor Dysfunct. 2001;12(5):308-11.

19.Tunn R, Petri E. Introital and transvaginal ultrasound as the main in the assessment of tool urogenital and pelvic floor dysfunctions: an imaging panel and practical approach. Ultrasound Obstet Gynecol. 2003;22(2):205-13.

20.Brandt FT, Albuquerque CDC, Arraes AF, Albuquerque $\mathrm{GF}$, Barbosa CD, Araújo CM. Influência do volume vesical na avaliação ultra-sonográfica da junção uretrovesical e uretra proximal. Radiol Bras. 2005;38(1):33-6.

21.Wise BG, Burton G, Cutner A, Cardozo LD. Effect of vaginal ultrasound probe on lower urethral tract function. Br J Urol. 1992;70(1):12-6.

22.Mouritsen L, Strandberg C, Frimodt-Moller C. Bladder neck anatomy and mobility: effect of vaginal ultrasound probe. Br J Urol. 1994;74(6):749-52.

23.Jimenez Cidre MA, Lopez-Fando Lavalle L, Quicios Dorado C, de Castro Guerin C, Fraile Poblador A, Mayayo Dehesa T. Ultrasound for the diagnosis of female urinary incontinente. Arch Esp Urol. 2006;59(4):431-9.

24.Haab F, Zimmern PE, Leach GE. Female stress urinary incontinence due to intrinsic sphincteric deficiency: recognition and management. J Urol. 1996;156(3):3-17.

25. Bhatia NN, Ostergard DR, McQuown D. Ultrasonography in urinary incontinence. Urology. 1987;29(1):90-4.

26.Fleischmann N, Flisser AJ, Blaivas JG, Panagopoulos G. Sphincteric urinary incontinence: relationship of vesical leak point pressure, urethral mobility and severity of incontinence. J Urol. 2003;169(3): 999-1002.

27.Bai SW, Chung KA, Rha KH, Kim SU, Park KH. Correlation between urodynamic test results, perineal ultrasound and degree of stress urinary incontinence. J Reprod Med. 2003;48(9):718-22. 